\title{
Re-visiting ICM theory and practice: Lessons learned from the Baltic Sea Region
}

Støttrup, Josianne Gatt; Dinesen, Grete E.; Janssen, Holger; Gillgren, Christina; Schernewski, Gerald

Published in:

Ocean \& Coastal Management

Link to article, DOI:

10.1016/j.ocecoaman.2017.02.002

Publication date:

2017

Document Version

Peer reviewed version

Link back to DTU Orbit

Citation (APA):

Støttrup, J. G., Dinesen, G. E., Janssen, H., Gillgren, C., \& Schernewski, G. (2017). Re-visiting ICM theory and practice: Lessons learned from the Baltic Sea Region. Ocean \& Coastal Management, 139, 64-76.

https://doi.org/10.1016/j.ocecoaman.2017.02.002

\section{General rights}

Copyright and moral rights for the publications made accessible in the public portal are retained by the authors and/or other copyright owners and it is a condition of accessing publications that users recognise and abide by the legal requirements associated with these rights.

- Users may download and print one copy of any publication from the public portal for the purpose of private study or research.

- You may not further distribute the material or use it for any profit-making activity or commercial gain

- You may freely distribute the URL identifying the publication in the public portal 


\section{Re-visiting ICM theory and practice: Lessons learned from the Baltic Sea Region}

Josianne G. Støttrup
jgs@aqua.dtu.dk
Grete E. Dinesen $^{\mathrm{a}}$
Holger Janßen
Christina Gillgren
${ }^{\mathrm{b}}$
Gerald Schernewski $^{\mathrm{b}}$,

aDTU-Aqua, Technical University of Denmark, National Institute of Aquatic Resources, Charlottenlund Castle, Jægersborg Allé 1, 2920, Charlottenlund, Denmark

${ }^{\mathbf{b}}$ Leibniz Institute for Baltic Sea Research (IOW), Seestrasse 15, 18119, Rostock-Warnemünde, Germany

${ }^{\mathbf{c} G i l l g r e n ~ a n d ~ A s s o c i a t e s, ~} 15$ Kent Street, Bicton, WA, 6157, Australia

dKlaipeda University, Marine Science and Technology Center, H. Manto 84, LT-92294, Klaipeda, Lithuania

${ }^{*}$ Corresponding author.

\section{Abstract}

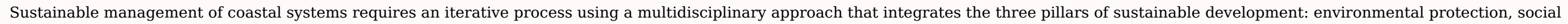

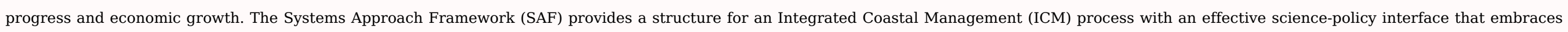

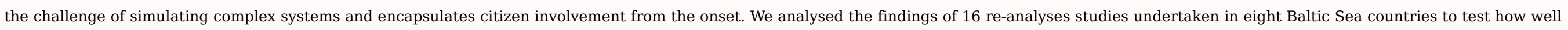

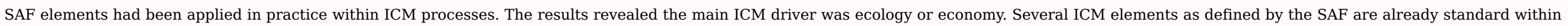

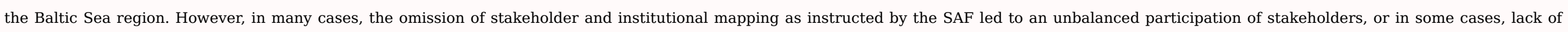

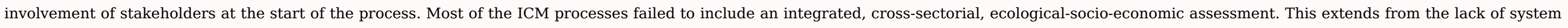

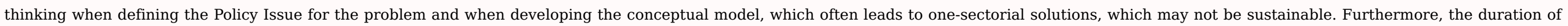

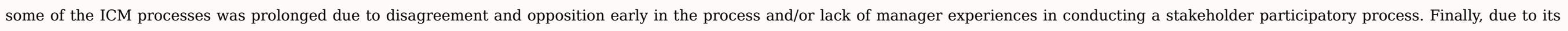
stringent structure the SAF was found to be a suitable quality assurance for sustainable ICM processes.

Keywords: Systems approach framework; Stakeholder engagement; Ecological-socio-economic assessment; Policy and science integration; Social ecology

\section{Introduction}

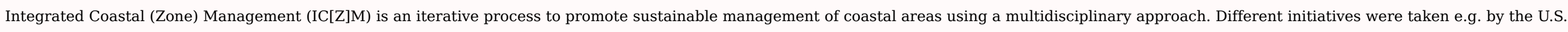

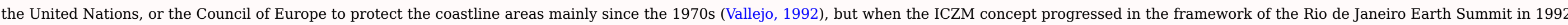

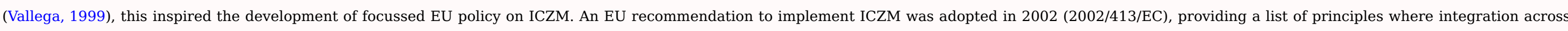

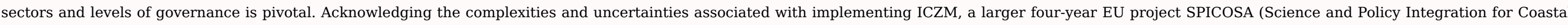

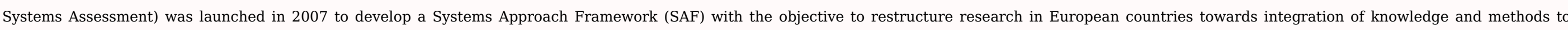

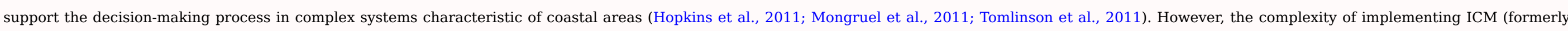




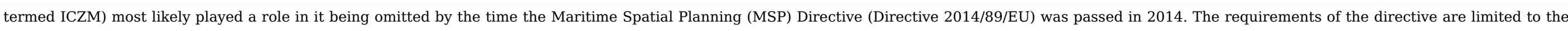
establishment and implementation of MSP by each coastal Member State, although it is also explicitly mentioned that land-sea interactions should be taken into account.

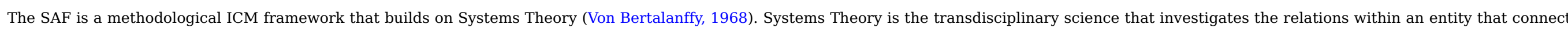

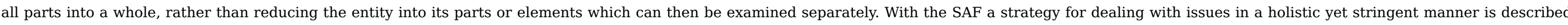

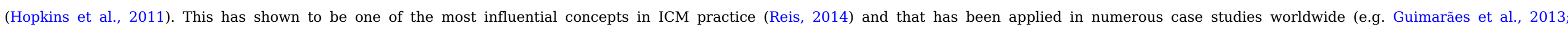

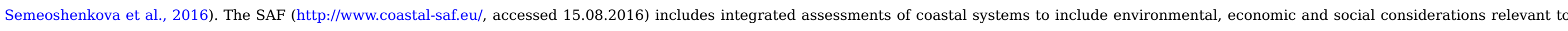

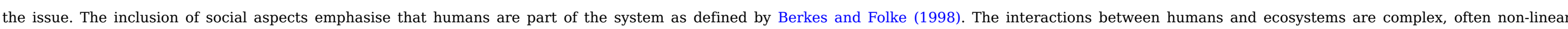

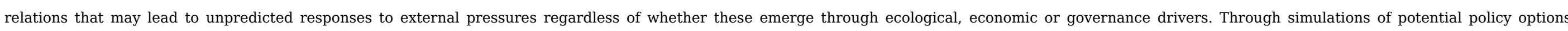

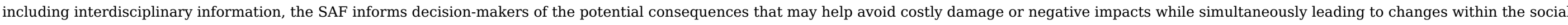
system. These changes may be in user perception of the ecological system or in interactions between different users.

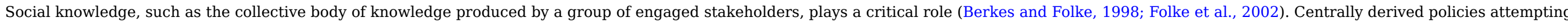

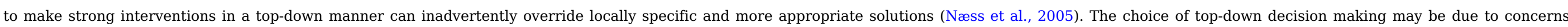

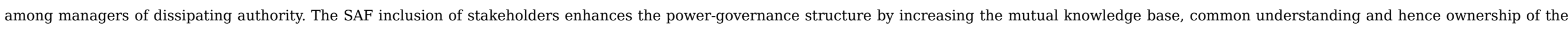
decision being made by managers and stakeholders working together (Mette, 2011).

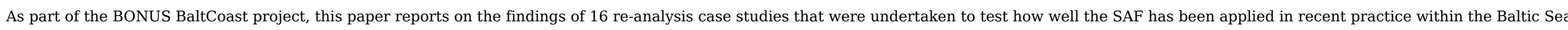

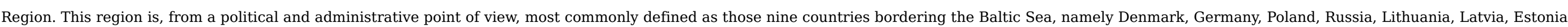

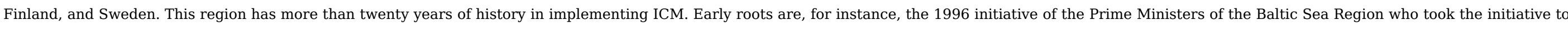

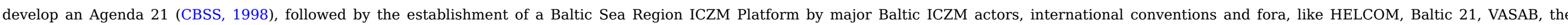

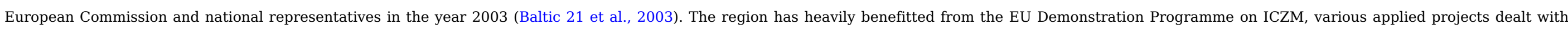

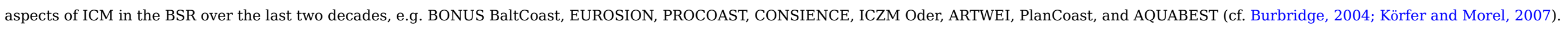

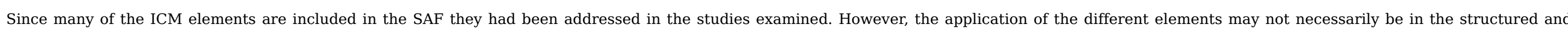

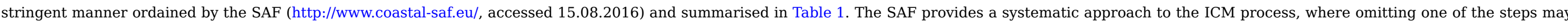

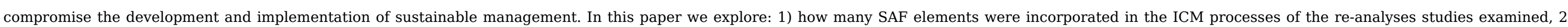

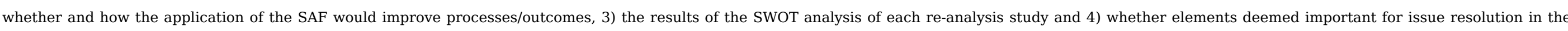
studies are absent in the SAF when re-analysed.

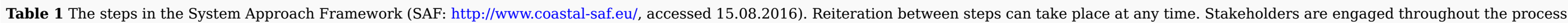
ESE: see list of abbreviations.

alt-text: Table 1

$$
\text { SAF step }
$$

SAF elements within each Step

Identify Policy Issue(s)

Mapping of Activities

Institutional mapping

Stakeholder mapping

List main ecosystem goods and services 
Data and analytical methods

Problem scaling

Develop ESE sub-models

Validation and calibration

System appraisal

Generating ESE Systems model

Calibration and validation and sensitivity tests

Preparing scenario simulations

System output

Running scenario simulations

Presenting results to stakeholders

Conduct stakeholder meeting and management options deliberations

\section{Methods}

\subsection{Case studies}

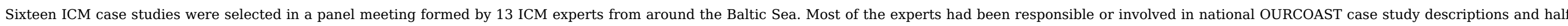

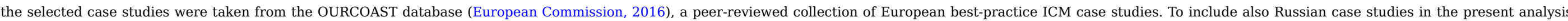

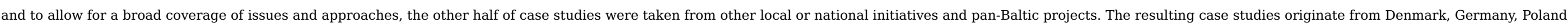

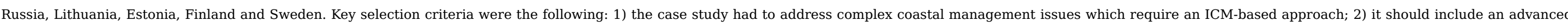

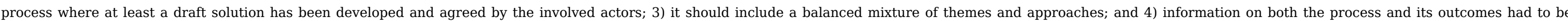
available, either as a written documentation or by access to more than one involved key person.

\subsection{The analytical approach}

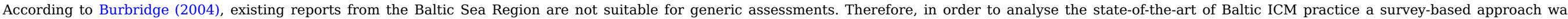

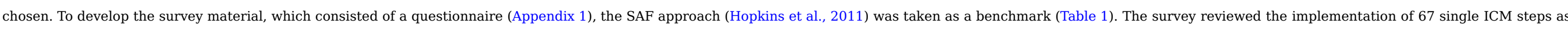

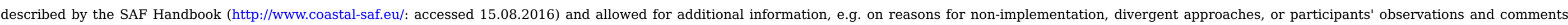

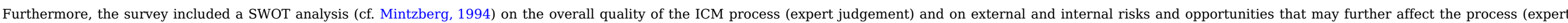

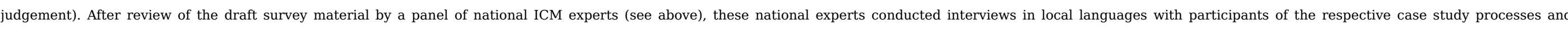
consolidated them by summing them up in one completed questionnaire for each case study. The reports were then reviewed and analysed by the authors of this paper.

\section{Results}

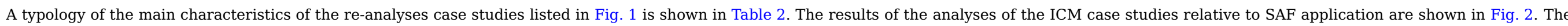

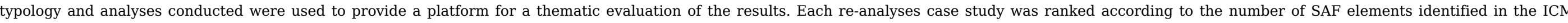
process and was independent of country (Fig. 3). The high ranking of the two Finnish examples is due to these being strategies and not addressing specific Policy Issues. 


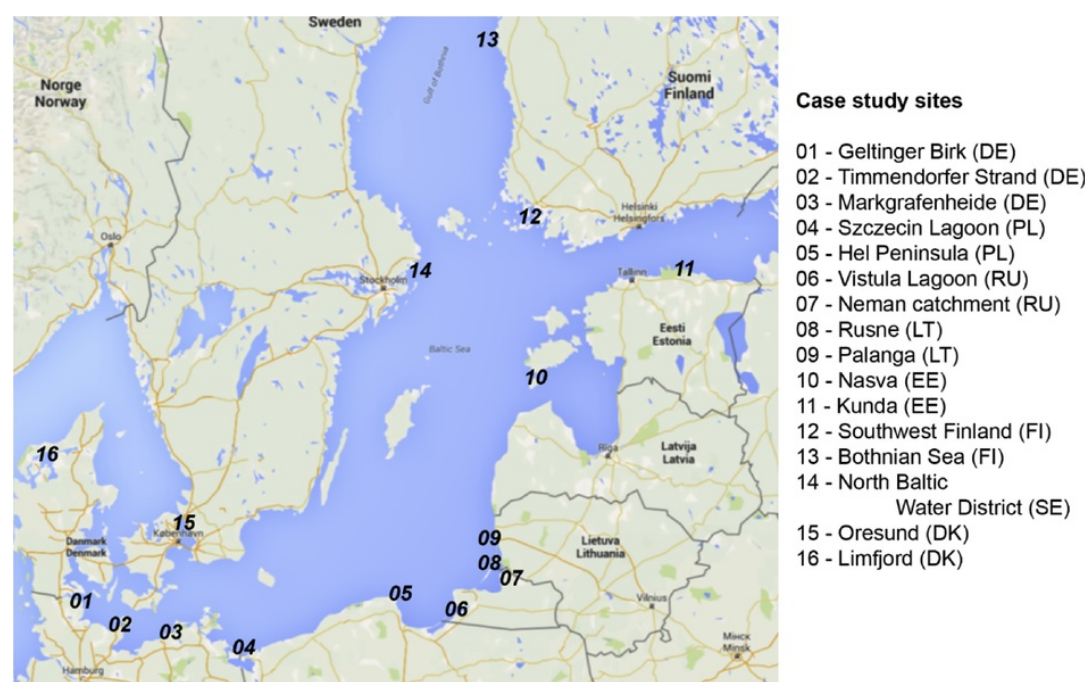

Fig. 1 The locations of the ICM cases re-analyzed indicated by name and listed on the left-hand side of the map of the Baltic Sea.

alt-text: Fig. 1

Table 2 Typology of the main characteristics of the re-analyses case studies.

alt-text: Table 2

\begin{tabular}{|c|c|c|c|c|c|c|c|c|c|c|c|c|c|c|}
\hline Rank & No & ICM case study & Country & $\begin{array}{l}\text { ICM process } \\
\text { initiated by }\end{array}$ & $\begin{array}{c}\text { ICM } \\
\text { driver(s) }\end{array}$ & $\begin{array}{l}\text { Issue type and } \\
\text { complexity }\end{array}$ & $\begin{array}{l}\text { Issue } \\
\text { identified at } \\
\text { outset }\end{array}$ & $\begin{array}{l}\text { Institutional } \\
\text { and } \\
\text { stakeholder } \\
\text { mapping }\end{array}$ & $\begin{array}{l}\text { The ICM team } \\
\text { composition }\end{array}$ & $\begin{array}{l}\text { Stakeholder } \\
\text { engagement }\end{array}$ & $\begin{array}{l}\text { Economic } \\
\text { assessment }\end{array}$ & $\begin{array}{l}\text { System } \\
\text { assessment } \\
\text { (ESE } \\
\text { model) }\end{array}$ & $\begin{array}{l}\text { Scenario } \\
\text { simulations }\end{array}$ & $\begin{array}{l}\text { ICM tin } \\
\text { scale }\end{array}$ \\
\hline 15 & 1 & $\begin{array}{l}\text { Coastal } \\
\text { realignment and } \\
\text { wetland } \\
\text { restoration } \\
\text { Geltinger Birk }\end{array}$ & Germany & $\begin{array}{l}\text { Federal state } \\
\text { ministry }\end{array}$ & $\begin{array}{l}\text { Ecological, } \\
\text { economical } \\
\text { (costs of } \\
\text { coastal } \\
\text { protection) }\end{array}$ & $\begin{array}{l}\text { Coastal protection \& } \\
\text { nature conservation }\end{array}$ & Yes & No mapping & $\begin{array}{l}\text { Authorities, local } \\
\text { managers \& } \\
\text { stakeholders }\end{array}$ & $\begin{array}{l}\text { Several } \\
\text { thematic } \\
\text { groups, } \\
\text { engaged } \\
\text { during } \\
\text { process }\end{array}$ & $\begin{array}{l}\text { Combined } \\
\text { cost analysis } \\
\text { of } \\
\text { restoration } \\
\text { and } \\
\text { protection }\end{array}$ & No & $\begin{array}{l}\text { No } \\
\text { modelling, } \\
\text { several } \\
\text { management } \\
\text { scenarios }\end{array}$ & $>20$ \\
\hline 9 & 2 & $\begin{array}{l}\text { Coastal } \\
\text { protection \& } \\
\text { wetland } \\
\text { restoration } \\
\text { Markgrafenheide }\end{array}$ & Germany & $\begin{array}{l}\text { Regional } \\
\text { authority }\end{array}$ & $\begin{array}{l}\text { Ecological, } \\
\text { economical }\end{array}$ & $\begin{array}{l}\text { Coastal protection \& } \\
\text { nature conservation }\end{array}$ & Yes & No mapping & $\begin{array}{l}\text { Local \& regional } \\
\text { authority } \\
\text { representatives }\end{array}$ & $\begin{array}{l}\text { Only public } \\
\text { participation } \\
\text { meetings }\end{array}$ & $\begin{array}{l}\text { Two } \\
\text { sectorial } \\
\text { cost } \\
\text { analyses }\end{array}$ & No & $\begin{array}{l}\text { Modelling of } \\
\text { coastal } \\
\text { evolution; no } \\
\text { alternative } \\
\text { scenarios }\end{array}$ & $>10$ \\
\hline 15 & 3 & $\begin{array}{l}\text { Coastal } \\
\text { protection } \\
\text { management: }\end{array}$ & Germany & $\begin{array}{l}\text { Federal state } \\
\text { ministry }\end{array}$ & $\begin{array}{l}\text { Economic } \\
\text { (flooding, } \\
\text { tourism) }\end{array}$ & $\begin{array}{l}\text { Coastal protection, } \\
\text { tourism \& urban } \\
\text { development }\end{array}$ & Yes & $\begin{array}{l}\text { No mapping, } \\
\text { multiple } \\
\text { stakeholders }\end{array}$ & $\begin{array}{l}\text { Local stakeholders } \\
\text { with moderator } \\
\text { (company }\end{array}$ & $\begin{array}{l}\text { Engaged at } \\
\text { outset of } \\
\text { process }\end{array}$ & $\begin{array}{l}\text { Sectorial } \\
\text { cost } \\
\text { analyses }\end{array}$ & Yes & $\begin{array}{l}\text { Yes, } \\
\text { qualitative } \\
\text { model }\end{array}$ & $>10$ \\
\hline
\end{tabular}




\begin{tabular}{|c|c|c|c|c|c|c|c|c|c|c|c|c|c|c|}
\hline & & $\begin{array}{l}\text { Timmendorfer } \\
\text { Strand \& } \\
\text { Scharbutz }\end{array}$ & & & & & & $\begin{array}{l}\text { and } \\
\text { institutions } \\
\text { identified }\end{array}$ & $\begin{array}{l}\text { contractedeontrated } \\
\text { by ministry) }\end{array}$ & & $\begin{array}{l}\text { (coastal } \\
\text { protection) }\end{array}$ & & $\begin{array}{l}\text { including all } \\
\text { ESE } \\
\text { components }\end{array}$ & \\
\hline 6 & 4 & Hel Peninsula & Poland & $\begin{array}{l}\text { National } \\
\text { managers }\end{array}$ & $\begin{array}{l}\text { Economic } \\
\text { (local } \\
\text { population } \\
\text { and } \\
\text { tourism) }\end{array}$ & $\begin{array}{l}\text { Climate change } \\
\text { (beach erosion) }\end{array}$ & Yes & No mapping & $\begin{array}{l}\text { National mangers } \\
\& \text { scientists }\end{array}$ & $\begin{array}{l}\text { Bilateral or } \\
\text { small } \\
\text { groups, } \\
\text { engaged } \\
\text { during } \\
\text { process }\end{array}$ & $\begin{array}{l}\text { One- } \\
\text { sectorial } \\
\text { cost analysis }\end{array}$ & $\begin{array}{l}\text { Bio- } \\
\text { economic } \\
\text { model }\end{array}$ & Yes & $>10$ \\
\hline 14 & 5 & $\begin{array}{l}\text { Szczecin lagoon - } \\
\text { Polish part }\end{array}$ & Poland & $\begin{array}{l}\text { Local } \\
\text { managers }\end{array}$ & Economic & $\begin{array}{l}\text { Climate change \& } \\
\text { Natura } 2000\end{array}$ & Yes & No mapping & $\begin{array}{l}\text { Local managers, } \\
\text { no formal team }\end{array}$ & $\begin{array}{l}\text { Bilateral or } \\
\text { small } \\
\text { groups, } \\
\text { engaged } \\
\text { during } \\
\text { process }\end{array}$ & $\begin{array}{l}\text { One- } \\
\text { sectorial } \\
\text { cost } \\
\text { analyses }\end{array}$ & No & No & $>10$ \\
\hline 1 & 6 & $\begin{array}{l}\text { Limfjord- } \\
\text { Denmark }\end{array}$ & Denmark & Scientists & Ecological & $\begin{array}{l}\text { EU WFD } \\
\text { implementation }\end{array}$ & Yes & Yes & $\begin{array}{l}\text { Scientists, } \\
\text { national } \\
\text { managers, } \\
\text { stakeholders }\end{array}$ & $\begin{array}{l}\text { Engaged at } \\
\text { outset of } \\
\text { process }\end{array}$ & $\begin{array}{l}\text { Cost- } \\
\text { effectiveness }\end{array}$ & Yes & $\begin{array}{l}\text { Yes - bio } \\
\text { economic } \\
\text { modelling, } \\
\text { multiple } \\
\text { cross } \\
\text { sectorial } \\
\text { scenarios }\end{array}$ & $<5$ \\
\hline 16 & 7 & Sound- Denmark & Denmark & Stakeholders & Economic & $\begin{array}{l}\text { Natural resource use } \\
\text { conflict }\end{array}$ & Yes & No mapping & No & No & No & No & No & $\begin{array}{l}<2.5 \text {, bu } \\
\text { unresolv }\end{array}$ \\
\hline 12 & 8 & $\begin{array}{l}\text { Restoration of } \\
\text { important } \\
\text { habitat through } \\
\text { sustainable } \\
\text { agricultural } \\
\text { practices, Rusne }\end{array}$ & Lithuania & $\begin{array}{l}\text { National } \\
\text { NGO }\end{array}$ & Ecological & $\begin{array}{l}\text { Nature conservation } \\
\& \text { sustainable } \\
\text { agricultural practises }\end{array}$ & Yes & $\begin{array}{l}\text { No mapping, } \\
\text { multiple } \\
\text { stakeholders } \\
\text { and } \\
\text { institutions } \\
\text { identified }\end{array}$ & $\begin{array}{l}\text { National NGO's, } \\
\text { local managers, } \\
\text { local stakeholders, } \\
\text { social scientists, }\end{array}$ & $\begin{array}{l}\text { Engaged at } \\
\text { outset of } \\
\text { process }\end{array}$ & No & $\begin{array}{l}\text { Partial } \\
\text { model. non- } \\
\text { quantitative } \\
\text { inclusion of } \\
\text { other ESE } \\
\text { components }\end{array}$ & $\begin{array}{l}\text { Ecological } \\
\text { scenarios }\end{array}$ & $<10$ \\
\hline
\end{tabular}




\begin{tabular}{|c|c|c|c|c|c|c|c|c|c|c|c|c|c|c|}
\hline 13 & 9 & $\begin{array}{l}\text { Integrated } \\
\text { shoreline } \\
\text { management for } \\
\text { a large harbour } \\
\text { city, Klaipeda } \\
\text { Seagate and an } \\
\text { adjacent seaside } \\
\text { resort Palanga }\end{array}$ & Lithuania & $\begin{array}{l}\text { National } \\
\text { managers }\end{array}$ & Economic & $\begin{array}{l}\text { Climate change } \\
\text { (beach } \\
\text { maintenance/tourism) }\end{array}$ & Yes & $\begin{array}{l}\text { No mapping, } \\
\text { multiple } \\
\text { stakeholders } \\
\text { and } \\
\text { institutions } \\
\text { identified }\end{array}$ & $\begin{array}{l}\text { National } \\
\text { managers, local } \\
\text { managers, } \\
\text { scientists, local } \\
\text { stakeholders, } \\
\text { NGO's }\end{array}$ & $\begin{array}{l}\text { Engaged at } \\
\text { outset of } \\
\text { process }\end{array}$ & No & No & $\begin{array}{l}\text { No - lack of } \\
\text { modelling } \\
\text { expertise }\end{array}$ & $<2.5$ \\
\hline 8 & 10 & $\begin{array}{l}\text { Cross-border } \\
\text { Neman River } \\
\text { Catchment - } \\
\text { Russian part }\end{array}$ & Russia & $\begin{array}{l}\text { National \& } \\
\text { local } \\
\text { managers }\end{array}$ & Ecological & $\begin{array}{l}\text { Water quality } \\
\text { protection }\end{array}$ & Yes & $\begin{array}{l}\text { No mapping, } \\
\text { multiple } \\
\text { institutions } \\
\text { identified }\end{array}$ & $\begin{array}{l}\text { National and local } \\
\text { managers, } \\
\text { scientists, } \\
\text { stakeholder } \\
\text { interests } \\
\text { addressed by } \\
\text { professionals }\end{array}$ & $\begin{array}{l}\text { Bilateral or } \\
\text { small } \\
\text { groups, } \\
\text { engaged } \\
\text { during } \\
\text { process }\end{array}$ & No & $\begin{array}{l}\text { Ecological } \\
\text { model } \\
\text { component }\end{array}$ & $\begin{array}{l}\text { Ecological } \\
\text { scenarios }\end{array}$ & \\
\hline 5 & 11 & $\begin{array}{l}\text { Vistula Lagoon - } \\
\text { comprehensive } \\
\text { management of a } \\
\text { water body }\end{array}$ & Russia & $\begin{array}{l}\text { National } \\
\text { managers, } \\
\text { cross-border } \\
\text { (Russia- } \\
\text { Poland) }\end{array}$ & $\begin{array}{l}\text { Ecological } \\
\& \\
\text { economic }\end{array}$ & $\begin{array}{l}\text { Water quality } \\
\text { protection }\end{array}$ & Yes & No mapping & $\begin{array}{l}\text { National } \\
\text { managers, local } \\
\text { managers, } \\
\text { scientists, } \\
\text { stakeholder } \\
\text { interests } \\
\text { addressed by } \\
\text { professionals }\end{array}$ & No & Yes & $\begin{array}{l}\text { Bio- } \\
\text { economic } \\
\text { modelling }\end{array}$ & $\begin{array}{l}\text { Economic } \\
\text { and } \\
\text { ecological } \\
\text { scenarios }\end{array}$ & $>10$ \\
\hline 10 & 15 & $\begin{array}{l}\text { The Järve-Nasva } \\
\text { case-study site } \\
\text { on Saaremaa } \\
\text { island - coastal } \\
\text { protection }\end{array}$ & Estonia & $\begin{array}{l}\text { Local } \\
\text { managers }\end{array}$ & Economic & $\begin{array}{l}\text { Climate change, HA } \\
\text { and nature } \\
\text { conservation }\end{array}$ & Yes & $\begin{array}{l}\text { No mapping, } \\
\text { multiple } \\
\text { stakeholders } \\
\text { identified }\end{array}$ & $\begin{array}{l}\text { Local managers, } \\
\text { local scientists, } \\
\text { local stakeholders, } \\
\text { NGO's }\end{array}$ & $\begin{array}{l}\text { Engaged at } \\
\text { outset of } \\
\text { process }\end{array}$ & No & No. & $\begin{array}{l}\text { Spatial } \\
\text { assessment } \\
\text { of human } \\
\text { activities }\end{array}$ & $>10$ \\
\hline 7 & 16 & $\begin{array}{l}\text { Kunda Port } \\
\text { development }\end{array}$ & Estonia & Stakeholders & Economic & $\begin{array}{l}\text { Coastal development, } \\
\text { resource use \& } \\
\text { nature conservation }\end{array}$ & Yes & $\begin{array}{l}\text { No mapping, } \\
\text { multiple } \\
\text { stakeholders } \\
\text { identified }\end{array}$ & $\begin{array}{l}\text { Port authorities, } \\
\text { other local } \\
\text { managers, nature } \\
\text { protection } \\
\text { representatives, } \\
\text { scientists, local } \\
\text { stakeholders }\end{array}$ & $\begin{array}{l}\text { Engaged at } \\
\text { outset of } \\
\text { process }\end{array}$ & $\begin{array}{l}\text { Yes - one- } \\
\text { sectorial } \\
\text { costs }\end{array}$ & $\begin{array}{l}\text { Ecological } \\
\text { models } \\
\text { (based on } \\
\text { historical } \\
\text { data) }\end{array}$ & $\begin{array}{l}\text { Scenarios } \\
\text { based on } \\
\text { historical } \\
\text { data }\end{array}$ & $<2.5$ \\
\hline
\end{tabular}




\begin{tabular}{|c|c|c|c|c|c|c|c|c|c|c|c|c|c|c|}
\hline 2 & 17 & $\begin{array}{l}\text { Coastal } \\
\text { management } \\
\text { strategy for } \\
\text { southwest } \\
\text { Finland }\end{array}$ & Finland & Scientists & $\begin{array}{l}\text { No ICM } \\
\text { driver }\end{array}$ & No issue identified & $\begin{array}{l}\text { ICZM/MSP } \\
\text { development }\end{array}$ & $\begin{array}{l}\text { No mapping, } \\
\text { multiple } \\
\text { stakeholders } \\
\text { identified }\end{array}$ & $\begin{array}{l}\text { Regional } \\
\text { managers, } \\
\text { scientists, multiple } \\
\text { stakeholders }\end{array}$ & $\begin{array}{l}\text { Engaged at } \\
\text { outset of } \\
\text { process }\end{array}$ & Yes & No. & $\begin{array}{l}\text { Spatial } \\
\text { assessment } \\
\text { of human } \\
\text { activities } \\
\text { and nature } \\
\text { resources } \\
\text { and values }\end{array}$ & n.a. \\
\hline 3 & 18 & $\begin{array}{l}\text { ICM in the } \\
\text { Bothnian Sea, } \\
\text { western Finland }\end{array}$ & Finland & Scientists & $\begin{array}{l}\text { No ICM } \\
\text { driver }\end{array}$ & No issue identified & $\begin{array}{l}\text { ICZM/MSP } \\
\text { development }\end{array}$ & $\begin{array}{l}\text { No mapping, } \\
\text { multiple } \\
\text { stakeholders } \\
\text { identified }\end{array}$ & $\begin{array}{l}\text { Regional } \\
\text { managers, } \\
\text { scientists, multiple } \\
\text { stakeholders }\end{array}$ & $\begin{array}{l}\text { Engaged at } \\
\text { outset of } \\
\text { process }\end{array}$ & Yes & No. & $\begin{array}{l}\text { Spatial } \\
\text { assessment } \\
\text { of human } \\
\text { activities } \\
\text { and nature } \\
\text { resources } \\
\text { and values. }\end{array}$ & n.a. \\
\hline 11 & 19 & $\begin{array}{l}\text { Implementation } \\
\text { of the WFD: The } \\
\text { North Baltic } \\
\text { Water District in } \\
\text { Sweden }\end{array}$ & Sweden & $\begin{array}{l}\text { National } \\
\text { managers }\end{array}$ & Ecological & $\begin{array}{l}\text { EU WFD } \\
\text { implementation }\end{array}$ & Yes & $\begin{array}{l}\text { No mapping, } \\
\text { multiple } \\
\text { stakeholders } \\
\text { and } \\
\text { institutions } \\
\text { identified }\end{array}$ & $\begin{array}{l}\text { National } \\
\text { managers, } \\
\text { scientists } \\
\text { consulted }\end{array}$ & $\begin{array}{l}\text { Engaged at } \\
\text { outset of } \\
\text { process }\end{array}$ & $\begin{array}{l}\text { Yes, cost- } \\
\text { effectiveness }\end{array}$ & $\begin{array}{l}\text { Partial } \\
\text { economic } \\
\text { assessment } \\
\text { at national } \\
\text { level. }\end{array}$ & No & \\
\hline
\end{tabular}




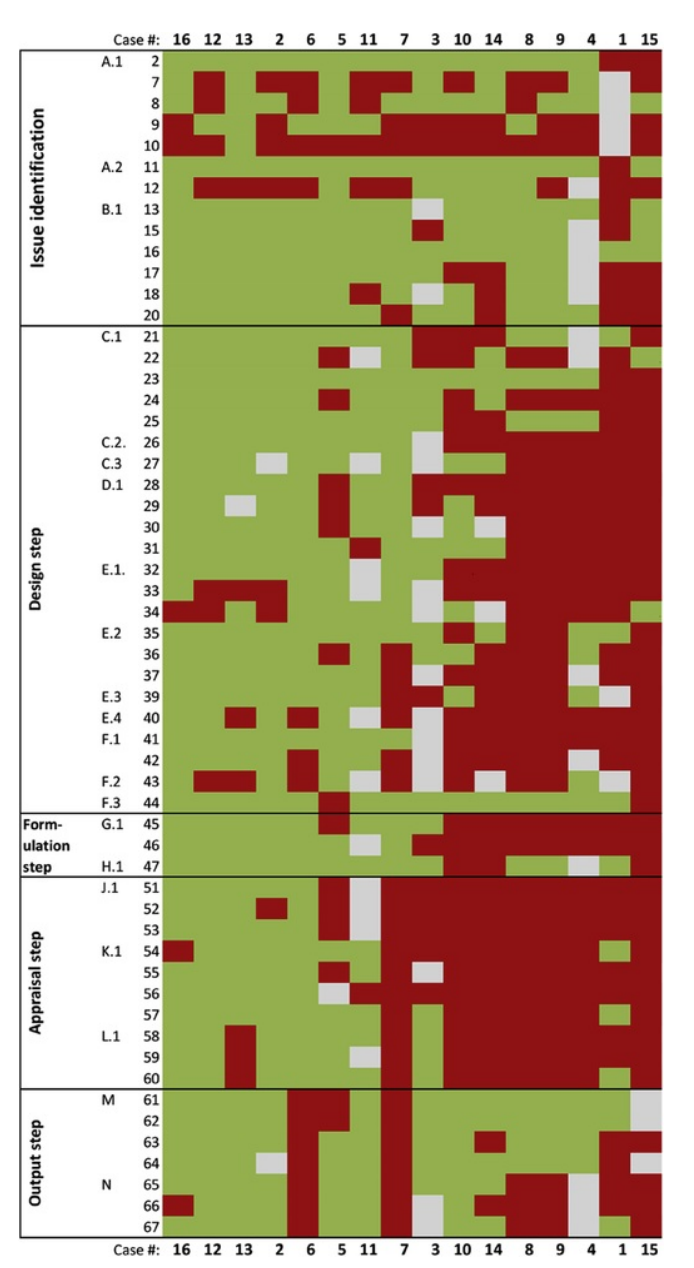

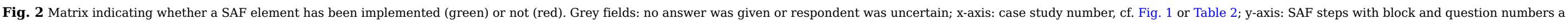

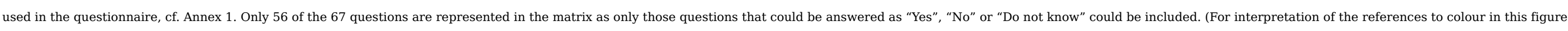
legend, the reader is referred to the web version of this article.) alt-text: Fig. 2

Rank per country

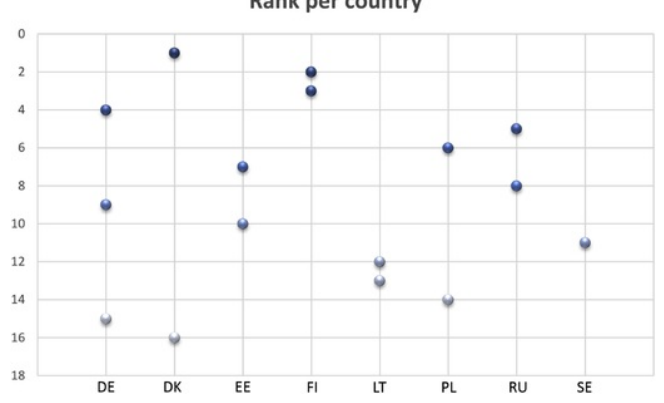




\subsection{Policy Issues and issue driver}

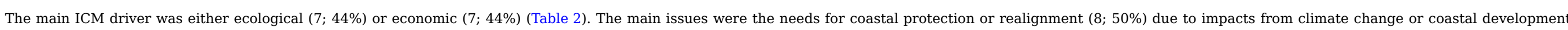

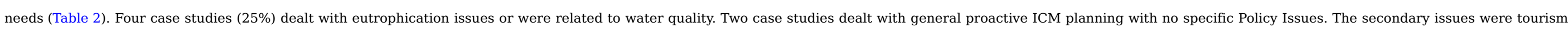

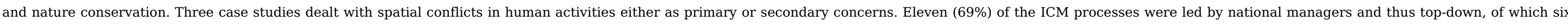

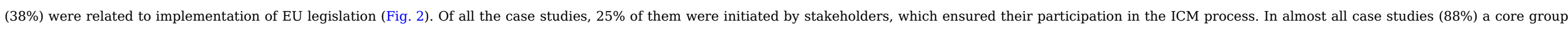
(Management Team) was established to deal with the Policy Issue (Fig. 2) but only in two of the cases was there knowledge of the SAF.

\subsection{Institutional and stakeholder mapping}

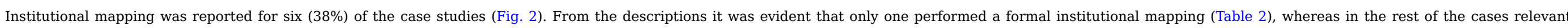

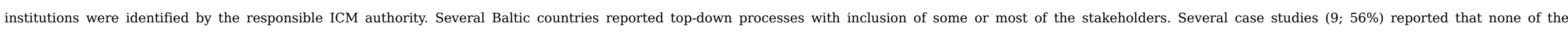

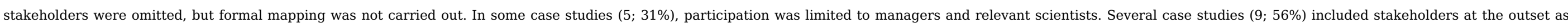
specified by the SAF, whereby stakeholders are involved in the formulation of the Policy Issue (Issue Identification).

\subsection{Background information relevant to the issue}

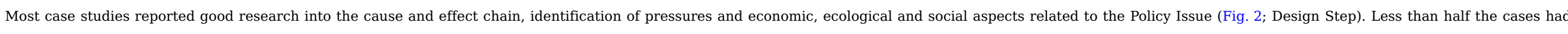

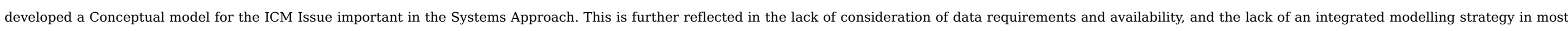
cases.

\subsection{Ecological-social-economic (ESE) modelling and scenario simulations}

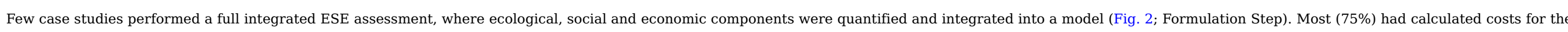

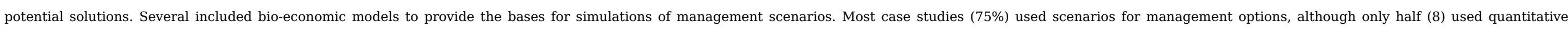
modelling methods for simulations of scenarios (Fig. 2; Appraisal Step).

\subsection{Stakeholder involvement in the decision process}

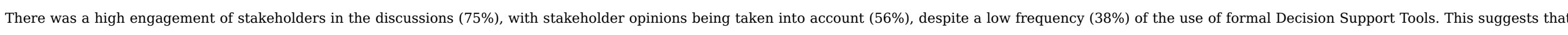

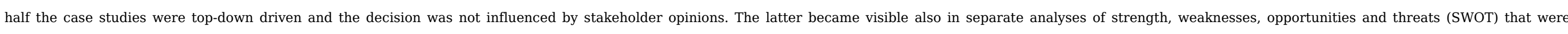

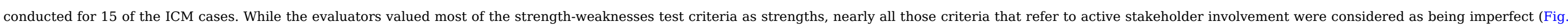
4). According to Fig. 2 there was a high engagement of stakeholders in the discussions (see above), but on average the reviewed case studies had some shortcomings in

- involving stakeholders in a review of ESE assessments,

- making use of local knowledge,

- active involvement of stakeholders,

- sufficient space for discussions, and in

- providing transparent results and decision making processes. 


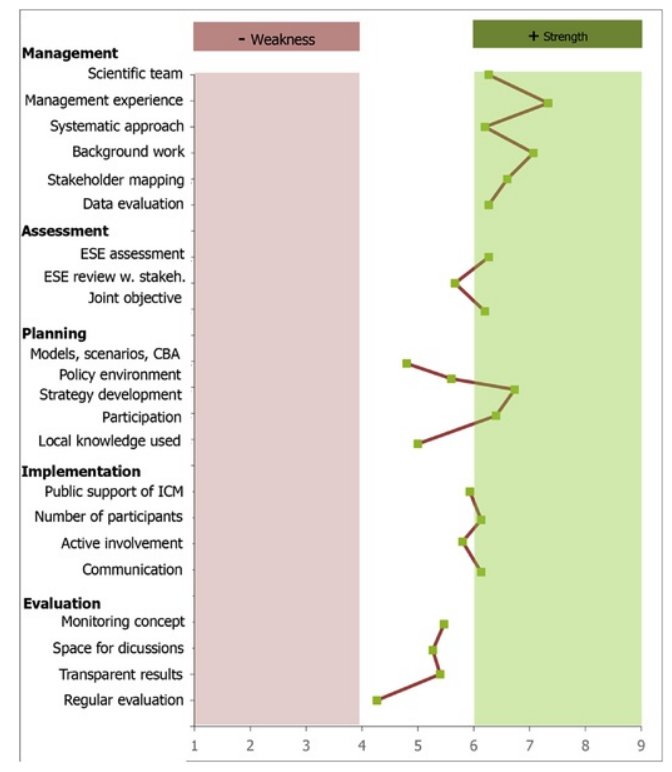

Fig. 4 Average values for strengths and weaknesses of 15 ICM case studies.

alt-text: Fig. 4

\subsection{External forcing and evaluation}

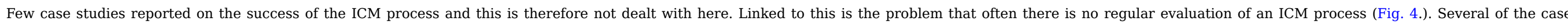

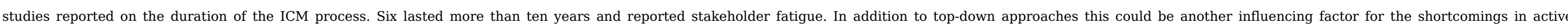

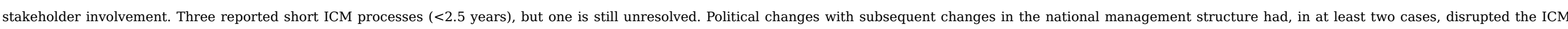
process or resulted in the lack of implementation of SAF results. An unsupportive policy environment was in many cases perceived as having negative impacts on the reviewed processes (Fig. 4).

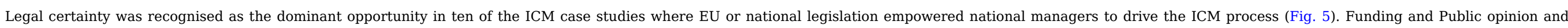

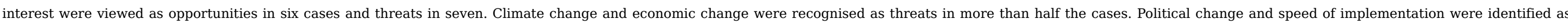
threats to the ICM process in the majority of the cases (12; 75\%). There were few responses to the potential threat or opportunity of institutional change ( 3 for each choice).

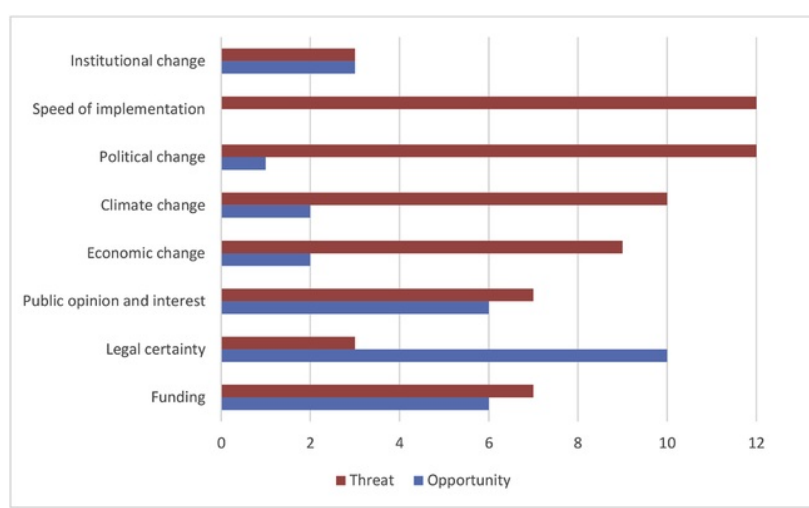

Fig. 5 Opportunities and threats by number of mentions. 


\section{Discussion and conclusions}

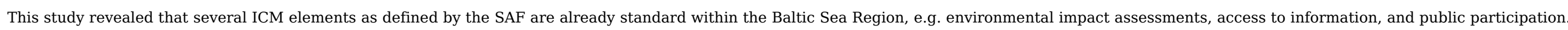

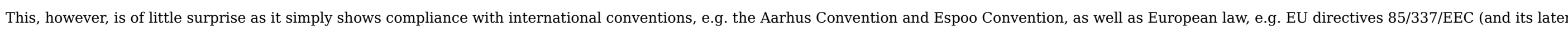

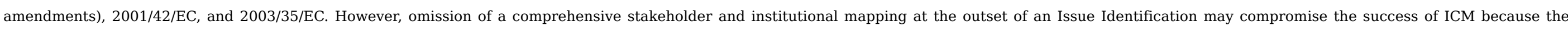

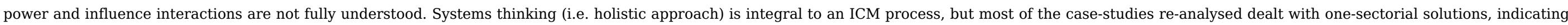
that holistic approaches were, in effect, rarely implemented. Furthermore, the duration of a successful ICM may need to be within a political timeframe.

\subsection{Systems thinking in ICM processes}

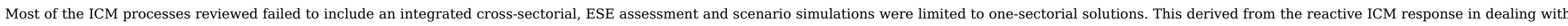

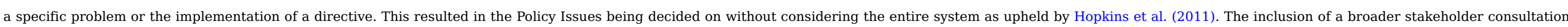

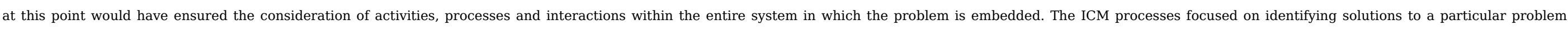

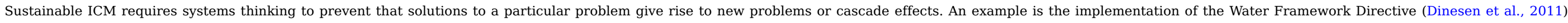

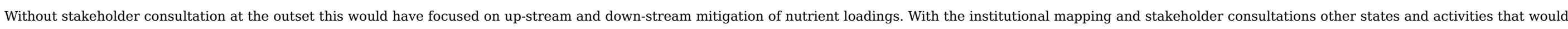
be affected by the solution were identified and included in the ESE assessment (Dinesen et al., 2011).

\subsection{Stakeholder engagement}

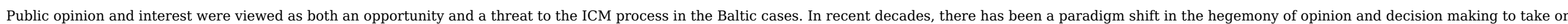

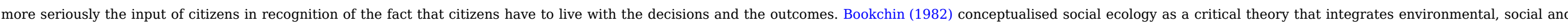
economic aspects for sustainable management. This has developed into a paradigm for sustainability thinking as demonstrated in Fig. 6 (Adams, 2006).

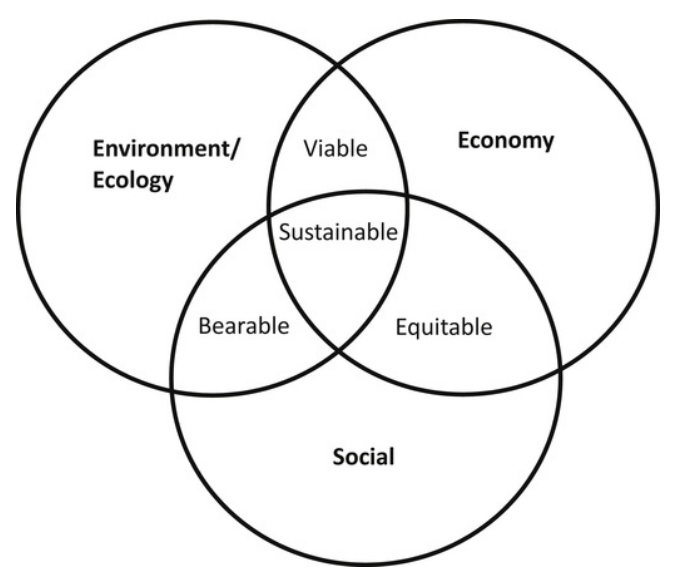

Fig. 6 The three pillars of sustainable development developed by Adams (2006), adapted by J. Dréo (https://commons.wikimedia.org/w/index.php?curid=1587372) and redrawn here. alt-text: Fig. 6

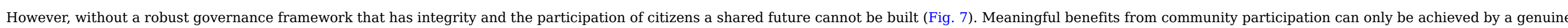

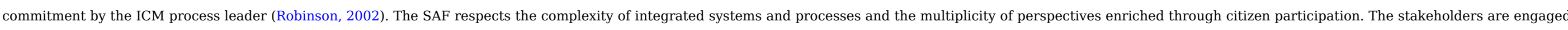

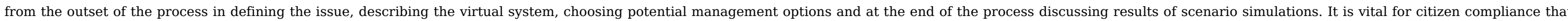

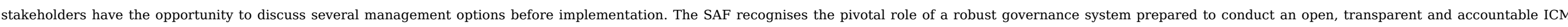




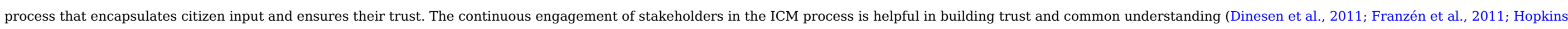

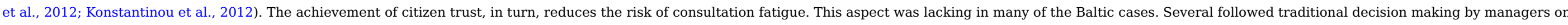
course of action, who to include, and public hearing to inform on decisions, thereby excluding the process of trust-building, mutual education and joint problem solving between managers and citizens.

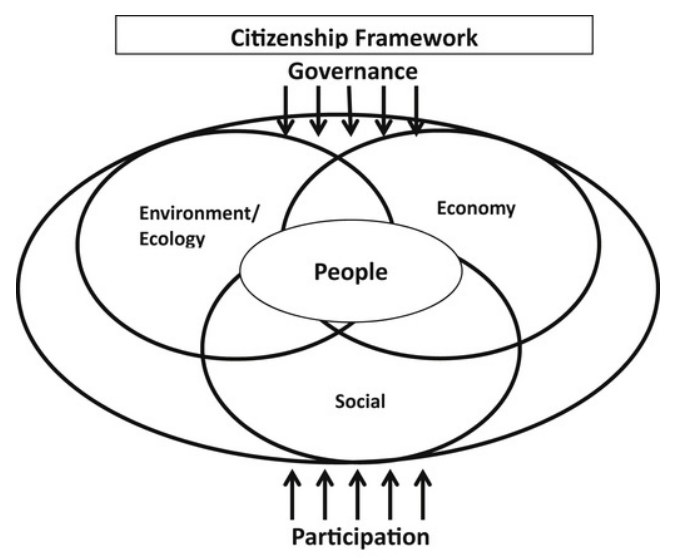

Fig. 7 The Citizenship Framework depicts how the three pillars of sustainability must be integrated with citizen participation and embedded in a robust governance framework. alt-text: Fig. 7

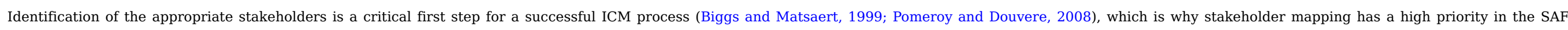

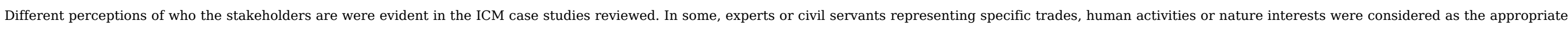

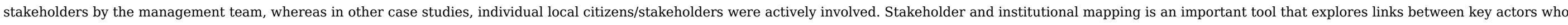

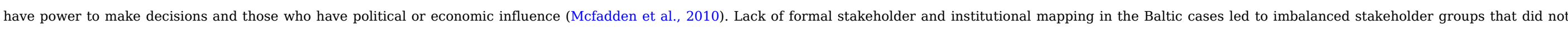

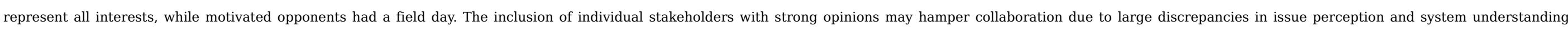

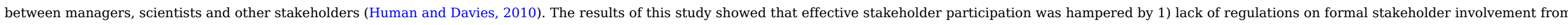
the onset of the process, 2) lack of experience by managers, and 3) lack of mapping and consultation tools.

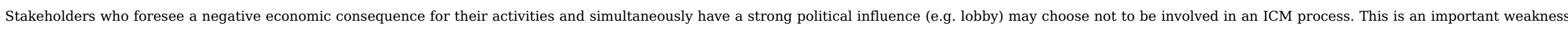

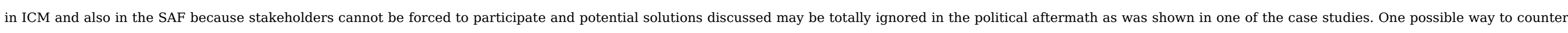

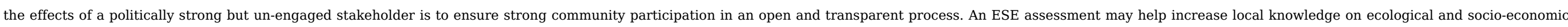

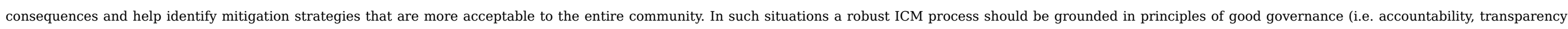
and openness). In very complex matters, it may be useful to employ consultants skilled in high social risk management to develop the appropriate methodology for engagement.

\subsection{Conceptual model and ESE assessment}

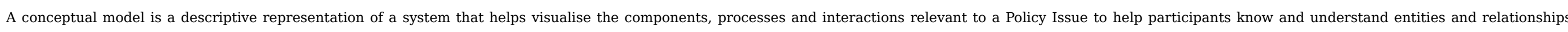

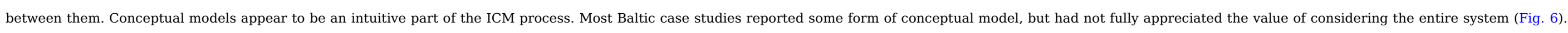

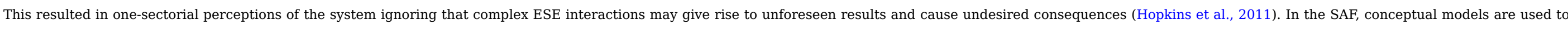

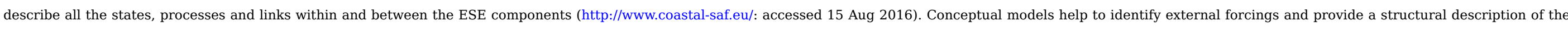

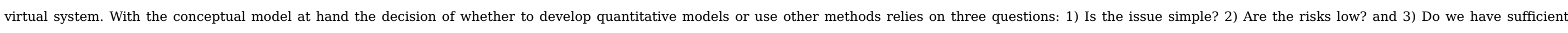
information to make reliable scenario simulations? If one can answer yes to all questions, development of a quantitative model may not be necessary. 


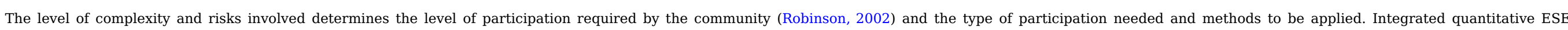

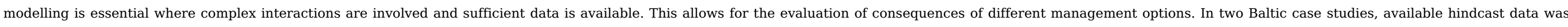

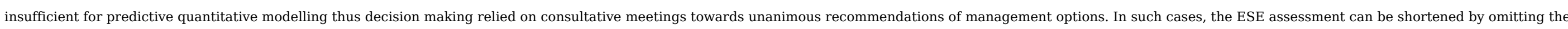

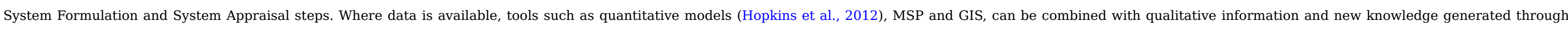

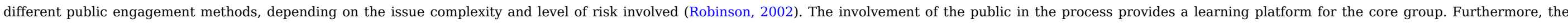

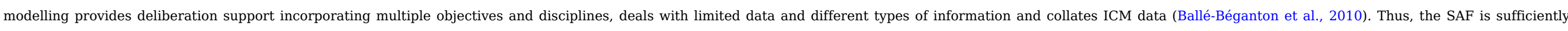
flexible to allow ESE assessments to be carried out at different information/data levels (Hopkins et al., 2012).

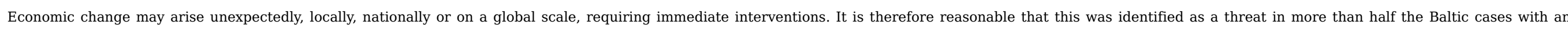

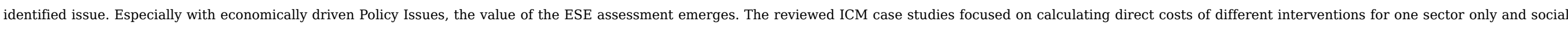

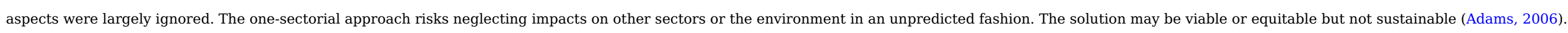

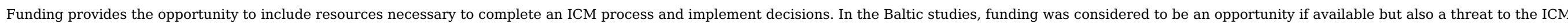

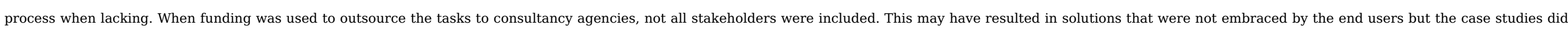
not provide information on this aspect.

\subsection{Legal certainty and implications of ICM not being in a legal framework}

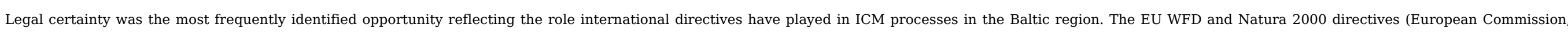

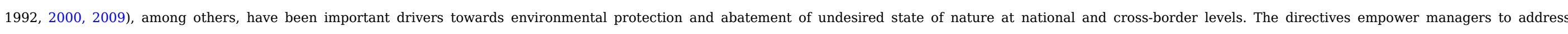
environmental issues but do not necessarily capture the need to 1) involve citizens until late in the process and 2) address cross-sectorial and multidisciplinary issues in an integrated approach.

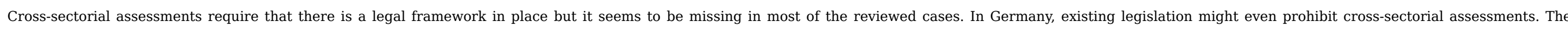

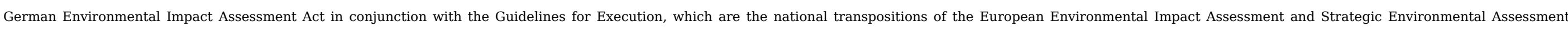

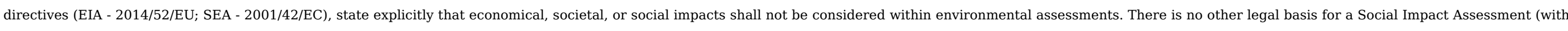
the exception of brown coal mining in North Rhine-Westphalia), wherefore social and economic impacts are often disregarded.

\section{In 2002, the EU recommended the implementation of ICM, providing a list of principles.(should not be a new paragraph here.)}

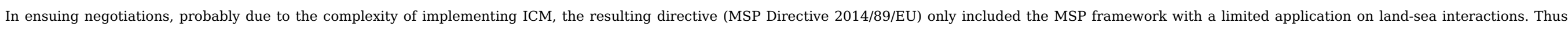

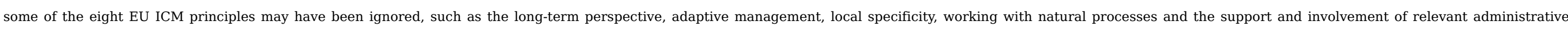
bodies. Sustainable growth in Europe would thus benefit from a legal driver towards a better quality in ICM processes.

\subsection{Process duration}

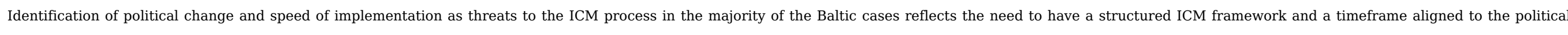

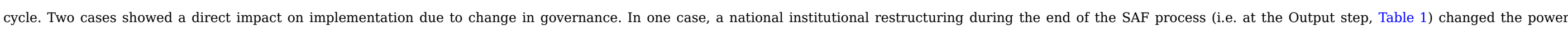

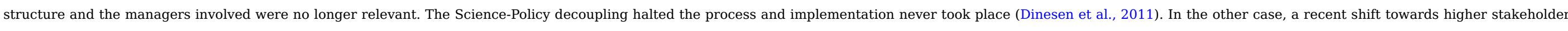

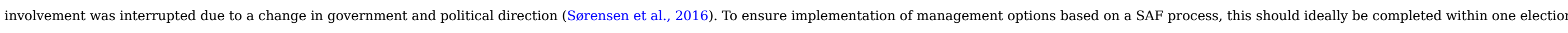

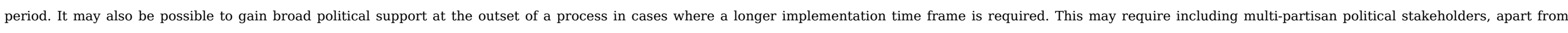
securing funding and resources for the complete process.

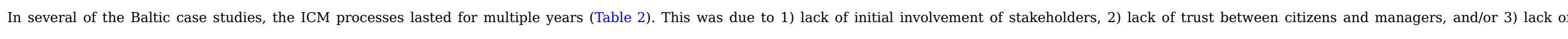

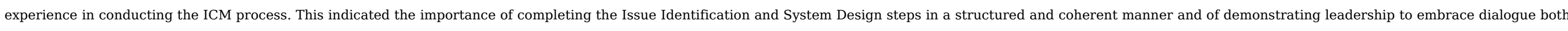




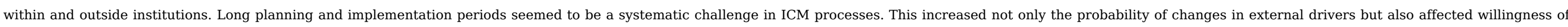

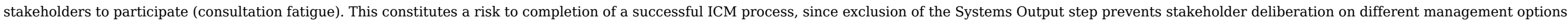

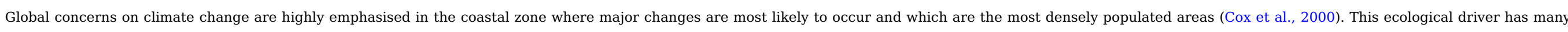

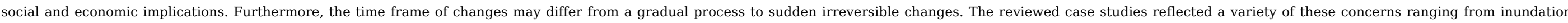

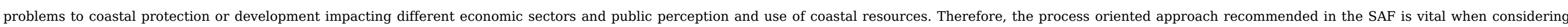
management solutions to climate change issues.

\subsection{Analytical shortcomings (reviewers based)}

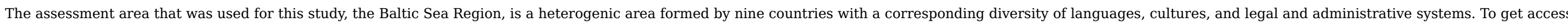

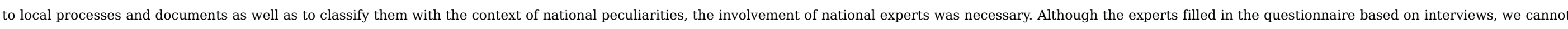

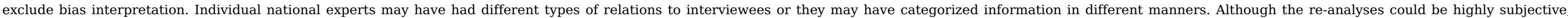
much of the extracted information from the questionnaires was coherent and provided a critical reflection on past ICM processes and where these can gain from SAF assimilation in future processes.

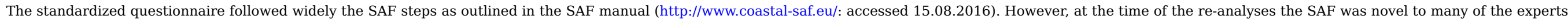

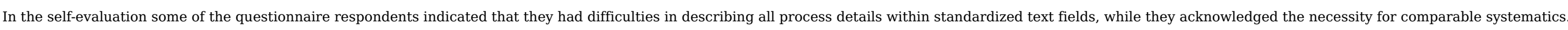

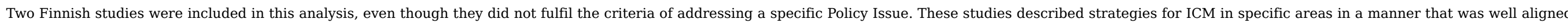

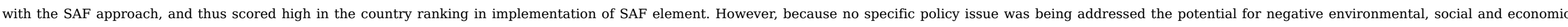

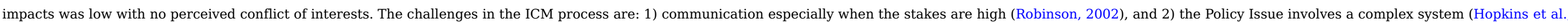
2012).

\subsection{SAF manual improvement}

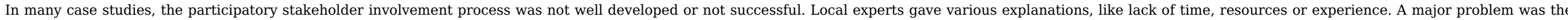

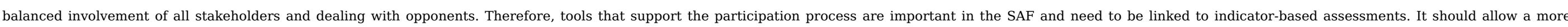
systematic, guided and thematically focussed stakeholder involvement process.

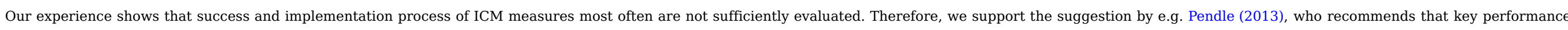

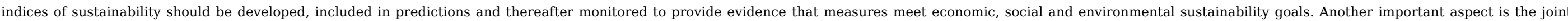

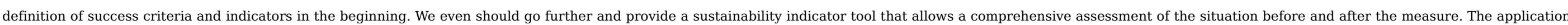

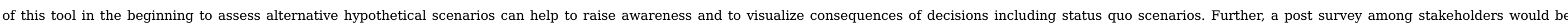

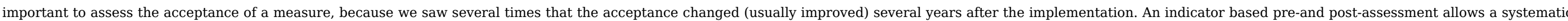
compilation of lessons learnt for future case studies and avoids repeating mistakes. However, it requires the provision of tools needed that allow fast assessment without detailed expert knowledge.

\subsection{Perspectives - how can SAF improve an ICM iterative process?}

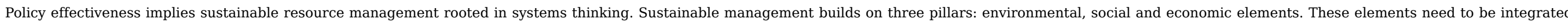

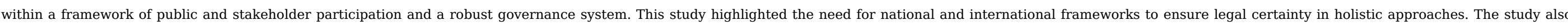

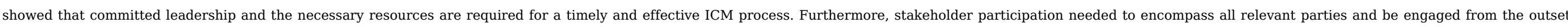

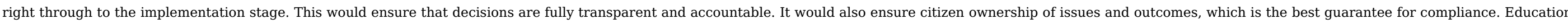

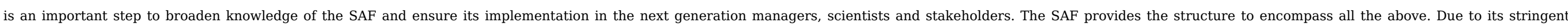
structure the SAF is a suitable quality assurance for sustainable ICM processes. 


\section{Acknowledgements}

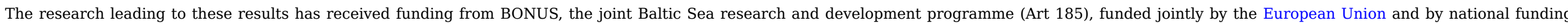

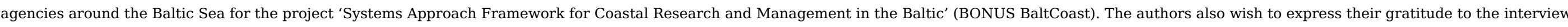

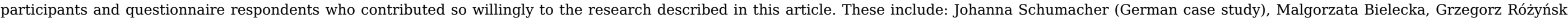

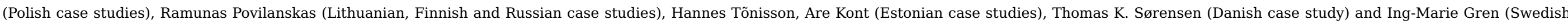
case study). We also thank two anonymous reviewers for their helpful comments.

\section{Appendix A}

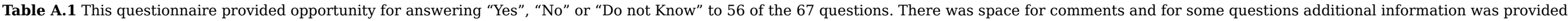
(here in brackets). The questions were based on the SAF handbook (http://www.coastal-saf.eu). The omitted questions were those requesting descriptive information on the case-study.

\section{alt-text: Table A.1}

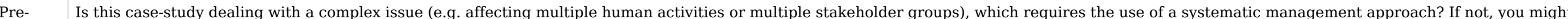

check wish to choose another case study.

\section{Issue Identification - getting started}

Was a management team built to lead the process and did it have scientific competences to some degree?

Was the issue initiated by EU directives?

Was the issue initiated by national regulations?

Was the issue voluntary initiated by stakeholders?

Were the team members familiar with SAF?

Issue Identification- preliminary study of the coastal zone

Were human activities (HA) and associated stakeholder groups determined?

Was a preliminary institutional map created to show up the relation between the governance and these HA and stakeholders?

\section{Issue Identification - reach agreement on the Policy Issue}

Was a reference group formed? (consisting of stakeholders, environmental managers and/or policy makers)

Was the issue discussed and agreed by the reference group?

Was background work done? (Analysis of available information on the cause-and-effect chain from HA to impact and evaluation of the importance of different HAs and impacts)

Where all possible measures and their costs and impacts identified?

Was equity, or allocation of effects among stakeholders, given any concern?

Were the main economic activities, the main ecosystem goods and services and the main economic drivers listed or mapped?

System Design - definition of the Virtual System

Was the cause-and-effect chain described? (Description of the chain from HA via ecosystem dysfunction to Impact that is involved in the problem)

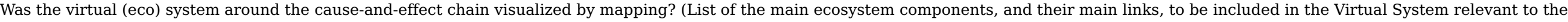
'Issue') 
Were the economic and social components included in the virtual system?

\section{C.2 System Design -identification of external hazards}

Were the external hazards listed which pose a risk for the real system in relation to the issue?

Was a synthesis of the system state made?

\section{System Design - construct conceptual models}

Was a method found to construct a model on the virtual system?

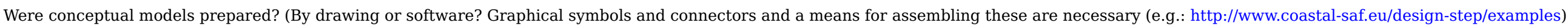

Was the reference group involved in developing conceptual models?

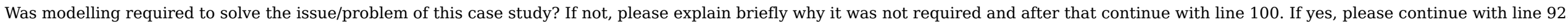

\section{System Design -identify model software, methods and formats}

Was a strategy for the modelling developed? (Were available models adapted? Were new sub-models for the virtual system simulation model developed?)

Were auxiliary models identified to be used?

Were other tools identified and used? (e.g. GIS or tools for statistical analyses)

\section{System Design - analysis of the economic dimensions of the Coastal Zone system and identification of suitable economic assessment methodologies}

Were costs calculated and compared for different actions?

Were assessments made of impacts on different stakeholders?

Were the economic dimensions of the models clear and explicit?

\section{System Design -acquire data}

Were relevant human activities identified and relevant 'pressure' or 'forcing' data acquired?

Was there a strategy for the issues of missing data and uncertainty?

\section{System Design -adjust the complexity of the Virtual System}

Was the complexity of the Virtual System adjusted (e.g. by focusing on core processes, by idealization, or by setting up a problem-oriented model)?

Was the feasibility of the implementation ensured?

\section{System Design - specification of formats for results}

Did the reference group think about a format for the presentation and visualizations of results?

\section{System Design -designed system report}

Was a technical reporting document compiled?

\section{System Formulation-modelling}

Was/were the model/models actually used with the case study work? 


\section{H.1 System Formulation-scenarios}

47 Were scenarios used to inform the case-study process and/or to run the models?

J.1 System Appraisal- preparation of the ESE models for coupling (ESE: Economic, Social, Ecological)

51 Were the ESE models checked concerning the appraisal objectives?

$52 \quad$ Were other links to other models or products of analyses integrated?

53 Were the ESE models run separately for purpose of interpretive analyses?

\section{K.1 System Appraisal-system simulations}

$54 \quad$ Were the priority and feasibility of scenarios reviewed and evaluated?

$55 \quad$ Were necessary input data for selected scenarios generated?

56 Were different scenario versions of the Simulation Model prepared, conducted and tested?

$57 \quad$ Were the results of scenarios documented?

L.1 System Appraisal-output preparation

$58 \quad$ Were the Hindcast and Scenario modelling results described and interpreted?

59 Were limits and liability of the model document within the analysis?

Were draft conclusions from the simulation analysis made and provided to stakeholders?

\section{System Output- hold a stakeholder forum}

Was a Stakeholder Forum organized?

Was a report of the forum drawn?

Were the results of scenarios/their interpretation discussed with stakeholders?

Were the outcomes of the stakeholder forum/hearing considered by the overall decision making process?

\section{System Output-deliberation}

Was a deliberation forum prepared?

Were decision support tools used for deliberation on the issue(s) of this case study?

Was the deliberation reflected by the stakeholder group/had participates the opportunity to add comments?

\section{Uncited references}

European Council, 1992

\section{References}

Adams W., The Future of Sustainability, 2006, 17, Rep. IUCN Renowned thinkers Meet. 29-31 January 2006 www.iucn.o.

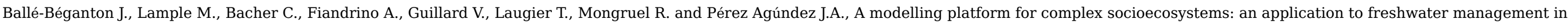
coastal zones, Model. Environ. Sake Proc. 5th Bienn. Conf. Int. Environ. Model. Softw. Soc. 2010 (2), 2010, 995-1002, iEMSs. 
Baltic 21, HELCOM and VASAB, Baltic Sea Region ICZM Platform Report 2003, 2003, (Helsinki).

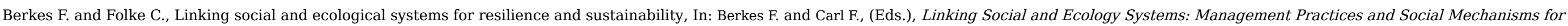
Building Resilience, 1998, Cambridge University Press; New York, 13-20.

Biggs S. and Matsaert H., An actor-oriented approach for strengthening research and development capabilities in natural resource systems, Public Adm. Dev. 19, 1999 , 231-262.

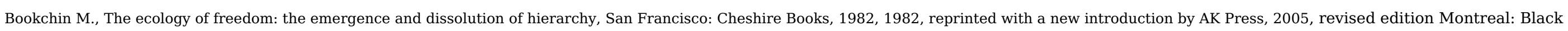
Rose Books, 1991.

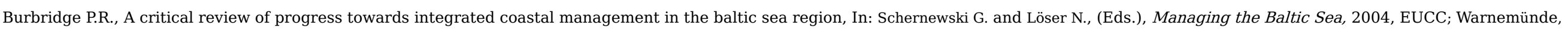

63-75, Coastline Reports 2.

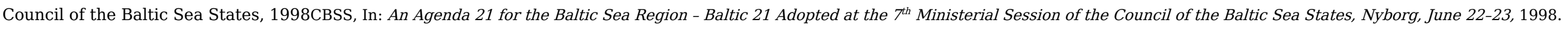

Cox P.M., Betts R.A., Jones C.D. and Spall S.A., Acceleration of Global Warming Due to Carbon-cycle Feedbacks in a Coupled Climate Model vol. 408, $2000,184-187$.

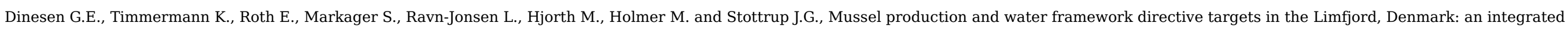
assessment for use in system-based management, Ecol. Soc. 16, 2011, http://dx.doi.org/10.5751/ES-04259-160426.

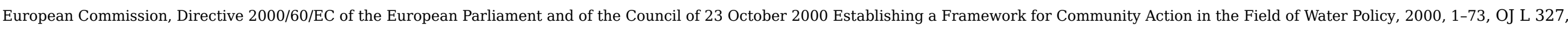
22.12.2000.

European Commission, Directive 2009/147/EC of the European Parliament and of the Council of 30 November 2009 on the Conservation of Wild Birds, 2009, 7-25, OJ L 20, 26.1.2010.

European Commission, OURCOAST - ICZM in Europe, 2016, (last assessed on 11 September 16) http://ec.europa.eu/environment/iczm/ourcoast.htm.

European Council, Council Directive 92/43/EEC of 21 May 1992 on the Conservation of Natural Habitats and of Wild Fauna and Flora, 1992.

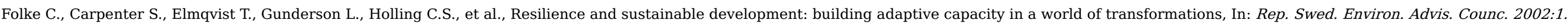
Minist. Environ., Stockholm, Swed, 2002.

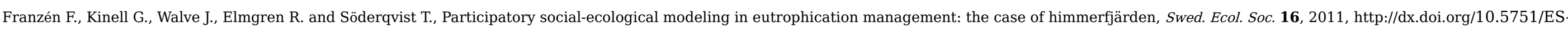
04394-160427.

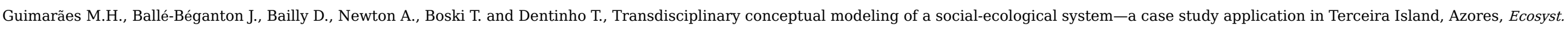
Serv. 3, 2013, 22-31.

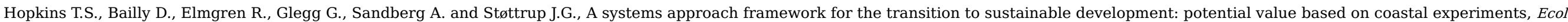
Soc. 17, 2012.

Hopkins T.S., Bailly D. and Støttrup J.G., A systems approach framework for coastal zones, Ecol. Soc. 16, 2011, http://dx.doi.org/10.5751/ES-04553-160425.

Human B.A. and Davies A., Stakeholder consultation during the planning phase of scientific programs, Mar. Policy 34, 2010, 645-654.

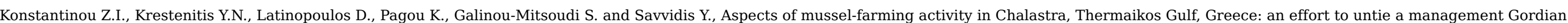
knot, Ecol. Soc. 17, 2012, http://dx.doi.org/10.5751/ES-04455-170101.

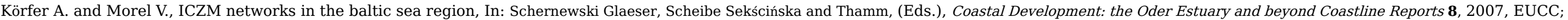

Warnemünde, 225-235 
Mcfadden L., Priest S. and Green C., Introducing institutional mapping: a guide for SPICOSA scientists, Spicosa Proj. Rep. 2010, 1-17.

Mintzberg H., The fall and rise of strategic planning, Harv. Bus. Rev. 72 (1), 1994, 107-114.

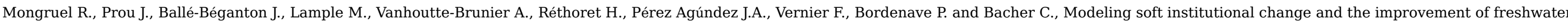
governance in the coastal zone, Ecol. Soc. 16, 2011, http://dx.doi.org/10.5751/ES-04294-160415, art15.

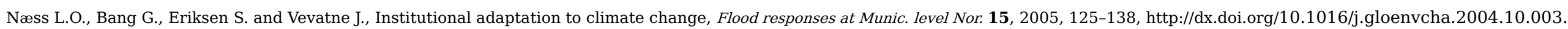

Pendle M., Estuarine and Coastal Managed Realignment Sites in England-A Comparison of Preditions with Monitoring Results for Selected Case Studies, 2013, HR Wallinford

Ltd http://eprints.hrwallingford.co.uk/664/.

Pomeroy R. and Douvere F., The engagement of stakeholders in the marine spatial planning process, Mar. Policy 32, 2008, 816-822.

Reis J., Introduction to systems approaches in coastal management-The legacy of the SPICOSA project, Mar. Policy 43, 2014, 1-2, http://dx.doi.org/10.1016/j.marpol.2013.03.012.

Robinson L., Public outrage and public trust, 2002

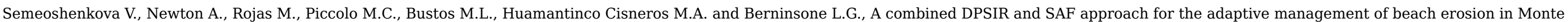
Hermoso and Pehuen Co (Argentina), Ocean Coast. Manag. 2016, http://dx.doi.org/10.1016/j.ocecoaman.

Sørensen T., Støttrup J. and Dinesen G., Best Practice Re-analysis: Denmark - Grains of Sand, a Sunken Treasure? vol. 25, $2016,8$.

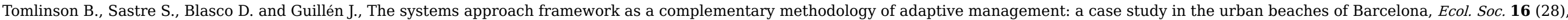
$2011 \mathrm{http} / / / \mathrm{dx}$. doi.org/10.5751/ES-04484-160428.

Vallega A., Fundamentals of Integrated Coastal Management, 1999, Kluwer Academic Publishers; Dordrecht, Netherlands, 269.

Vallejo S.M., Integrated Marine Policies: Goals and Constraints, In: Fabbri P., (Ed), Ocean Management in Global Change, 1992, Elsevier; London, 153-168.

Von Bertalanffy L., General System Theory vol. 1, 1968, Georg. Braziller; New York, 289.

\section{Highlights}

- The SAF is highly suitable as quality assurance for sustainable ICM

- Omitting stakeholder and institutional mapping may compromise the success of ICM.

- Several reviewed ICM processes were not holistic in their approach.

- With SAF an ICM process may run more effectively, within a political timeframe.

- Quality in ICM processes would benefit from the establishment of formal directives.

\section{Queries and Answers}

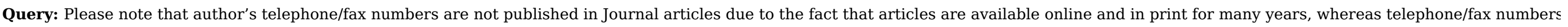
are changeable and therefore not reliable in the long term.

Answer: OK 


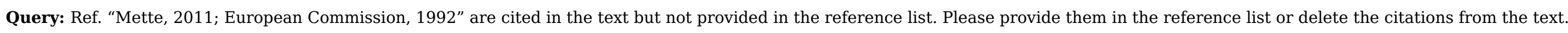

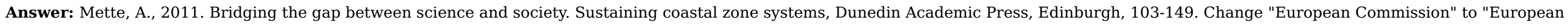
Council" in the reference. Ref: European Council,1992. Council Directive 92/43/EEC of 21 May 1992 on the conservation of natural habitats and of wild fauna and flora.

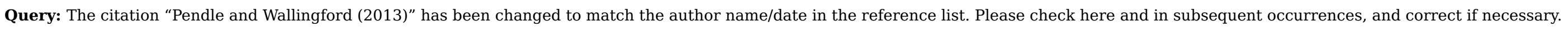
Answer: this should be: Pendle, M., Wallingford, H, R., (2013). Estuarine and coastal managed realignment sites in England selected case studies. HR Wallingford, 1-36. http://eprints.hrwallingford.co.uk/664/1/HRPP627-Managed realignment.pdf. Needs to be corrected in the reference list and in the reference.

Query: Could you please provide the grant number for European Union, if any?

Answer: there is no grant number for this grant. We only refer to the project name: BONUS BaltCoast.

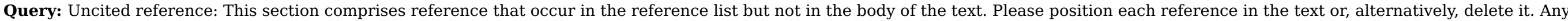
reference not dealt with will be retained in this section. Thank you.

Answer: As you can see from the answer to Q2, it should be left in as the reference in the text was wrong.

Query: Please confirm that given names and surnames have been identified correctly.

Answer: Yes all correct.

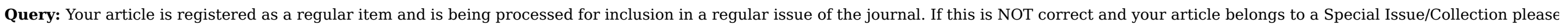
contact j.aranha@elsevier.com immediately prior to returning your corrections.

Answer: Yes 